\title{
Wireless sensor networks in a maritime environment
}

\author{
W. Kavelaars* and M. Maris \\ TNO Defence, Security and Safety \\ P.O. 96864, $2509 \mathrm{JG}$ \\ The Hague, The Netherlands
}

\begin{abstract}
In the recent years, there has been a lot of research on sensor networks and their applications. In particular for monitoring large and potentially hostile areas these networks have proven to be very useful. The technique of land-based sensor networks can be extrapolated to sensing buoys at sea or in harbors. This is a novel and intriguing addition to existing maritime monitoring systems. At TNO, much research effort has gone into developing sensor networks. In this paper, the TNOdes sensor network is presented. Its practical employability is demonstrated in a surveillance application deploying 50 nodes. The system is capable of tracking persons in a field, as would be the situation around a military compound. A camera-system is triggered by the sensors and zooms into the detected moving objects. It is described how this system could be modified to create a wireless buoys network. Typical applications of a wireless (and potentially mobile) buoy network are bay-area surveillance, mine detection, identification and ship protection.
\end{abstract}

Keyword: Sensor networks, force protection, buoys.

\section{INTRODUCTION}

Autonomous surveillance in harbors and at sea is a thriving field of research. This is because it is a security necessity to gather situational awareness about the environment in order to assess potential threats. Current sensor suites at sea in the form of buoys are typically standalone systems that communicate through satellite with a central station. Hence, for autonomous surveillance of a large area, they are not very suited.

Recently, a novel technology is being developed, which potentially offers true autonomous surveillance. This technology is called "wireless sensor networks". There is much research being conducted currently with respect to this topic. Most developed systems have found applications in land-based surveillance or monitoring tasks [1][2][3].

We envision that this technology can be easily ported to a marine environment, deploying mobile buoy networks that can maneuver and form a local intelligent network. Hence, such buoy networks can be placed anywhere while given a certain task to accomplish. The buoys can be dropped by an airplane or submerged from a submarine in the area that needs to be monitored. The buoys will autonomously adopt their position corresponding to their task in the network. Besides areamonitoring, typical tasks include protection of (military) assets, such as ships, harbors and oil-platforms, and minedetection.

In this paper, we will first describe the principle of a sensor network. TNO has developed its own sensor networks platform which forms the base of applications involving autonomous monitoring, as introduced in section 2 of this document. For the purpose of demonstration, a monitoring system was developed, tracking persons in a large area. This system is presented in section 3. Furthermore, its use in a maritime environment is elaborated in section 4. Conclusions are presented in the final section.

\footnotetext{
*winelis.kavelaars@tno.nl
} 


\section{WIRELESS SENSOR NETWORKS}

At TNO, research has focused on developing a computing concept using cooperative devices which share knowledge and negotiate about observations and resources to accomplish a given task. This work has resulted in numerous applications. A notable one is a prototype of an autonomous cooling system on Navy ships [4]. The advantage of inter-device communication is that better extraction of locally available information can be achieved. Particularly when sharing information between devices, the reliability of assessments of events in the world can be greatly improved. Therefore, the devices implicitly posses the capability to provide human operators with proper situational awareness and support decision making processes.

This work has resulted in the creation of TNOdes ${ }^{\mathrm{TM}}$ (TNO Distributed Embedded Systems), see Figure 1 (left). One TNOdes is a basic component containing data-processing and radio-communication facilities. In principal, these are the basic functions that are always required in a multi-device network. On top of that, the application specific layer is implemented. Hence, some TNOdes will be augmented with a sensor plus related information processing, while others are enhanced with an actuator, plus processing. This has resulted in the SOWNET concept (see Table 1) as a framework for cooperative information processing using small sensor nodes. The challenge of information extraction is to optimize the limited power/performance ratio in these devices.
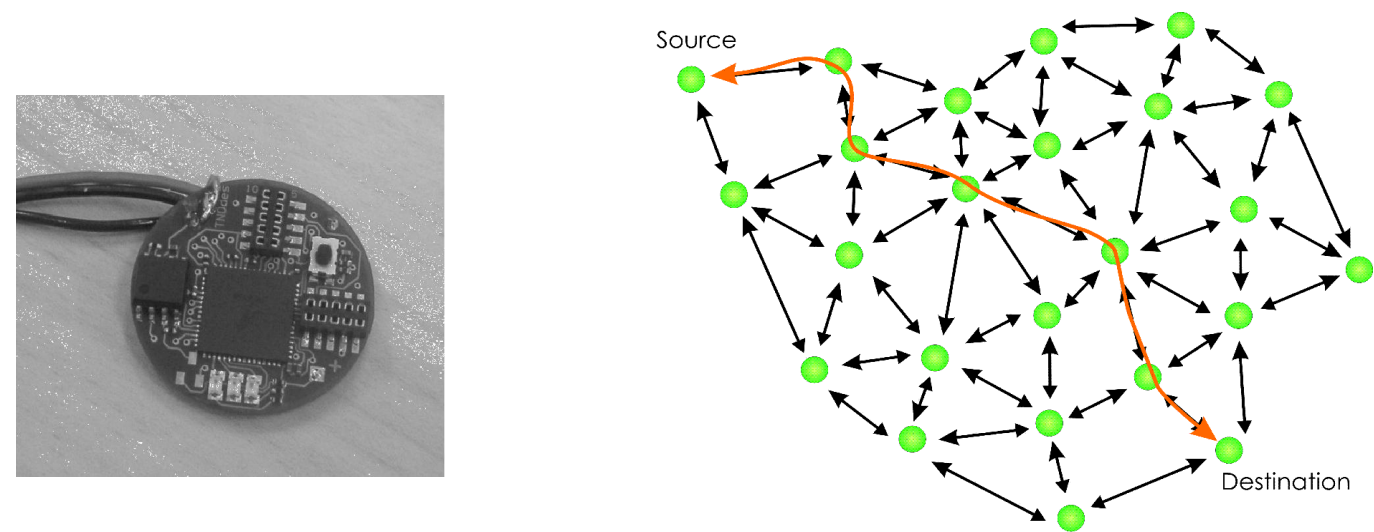

Figure 1. TNOdes component (left). Typical routing scheme throughout a sensor network. It shows that sensor data doesn't need to be sent directly from the "source" to the "destination", but can use a number of "hubs" to get there.

Table 1- Characteristics of SOWNet ${ }^{\mathrm{TM}}$ and TNOdes ${ }^{\mathrm{TM}}$ (C) TNO)

\begin{tabular}{|l|l|}
\hline SOWNet & TNOdes \\
\hline - Autonomous & - Autonomous \\
- Ad hoc (quickly deployable) & - Low cost \\
- Scalable & - Small \\
- Efficient use of nodes & - Light weight \\
- Data routing & - Low Power \\
- Data aggregation & - Limited communication \\
- distributed intelligence & capacity \\
- Redundancy & - Limited intelligence \\
- Geographical information & - Task specialization \\
- Robust & - Sensor/actuator \\
- Secure and safe & \\
\hline
\end{tabular}


A wireless sensor network is a self-organizing network consisting of numerous small, self-sustaining and intelligent sensor nodes. A node consists of one of more sensors, a battery, a radio transceiver and an embedded processor with dedicated software. The size of a node is generally determined by the application, the sensors to be used, the antenna, the power requirements and the battery size. Due to technological progress in integrated, low-power communication devices, current nodes can be made very small, not larger than a golf ball.

A typical usage of a wireless sensor network is to deploy many small and intelligent devices rather than one (powerful) sensor, e.g. to monitor a particular area. This may be done by virtually dividing the whole area of interest into small subareas and using one sensor node to cover a sub-area. Due to the embedded processor and capability of inter-node communication, the nodes are able to interpret sensor data and share information with each other locally for better interpretation of occurring events in their area. This functionality enables local decision making and possibly the initiation of local autonomous counter-measures. The level of autonomy and local decision making typically depends on the Rules of Engagement and the wishes of the commanding officers.

In a wireless sensor network, the sensor data does not have to be sent directly to a remote base station. Such is typically done in a centralized network. Instead, data can be sent to the base station by relaying through other nodes in a distributed ad-hoc fashion, see Figure 1 (right). Another approach is to use local gateways that collect data from a few nodes and relay the data further. This enables the use of low power nodes that need to transmit only over a short distance, resulting in smaller, low cost nodes with potentially longer life-times.

Hence the basic idea of a sensor network is to use a number of low-cost sensor-nodes to perform the same task as a single expensive sensor-system. This method also increases the reliability of the alarm system, since many paths lead to the control station (e.g., a ship surrounded by intelligent buoys). In a single-sensor system, destroying the sensor results in total system-breakdown. In a (distributed) sensor network, the loss of one sensor only means the loss of a small part of the system whose functionality may be taken over by other nodes (graceful degradation). Furthermore, a wireless sensor network can operate without the supervision of personnel (the latter may be desired in case suspicious activities are detected). In addition, decreasing the area to be covered by one sensor-node means that the corresponding sensor-node can lower its energy consumption because it doesn't have to transmit its data as far, assuming that more sensor-nodes are used to cover the entire area.

The nodes in a wireless sensor network are battery powered and therefore energy-constrained. In order to maximize a node's lifetime, special care must be taken in the hardware and software design. Once the system has been manufactured, additional energy savings can be achieved by using energy-efficient algorithms and protocols. TNO has developed a network protocol wherein the lifetime of a node can be vastly extended. This leads to a TNOdes lifetime of many months, hence useful for military applications. The methods that are used to accomplish this include shutting off the radio transceiver when no communication is required. Furthermore, the use of a dedicated node as gateway, consuming more energy than the surrounding nodes, also decreases the energy usage per node. Therefore, the nodes in the network don't have to be constantly active. Instead, they go into sleep-mode as long as nothing interesting is happening. The nodes are event driven, meaning that they are woken up by an event triggered by the node's sensor which stays active all the time. Note that many types of sensors consume much less energy than a processor or a radio-transmitter.

\section{AUTONOMOUS SURVEILLANCE SYSTEM}

To evaluate the sensing concept described in the previous sections, an experimental monitoring system was developed. The chosen application is compound security. This is because of the priority given by the Defense department to this topic. Compound security is an important issue in peace-keeping operations as the military force is subject to many types of relatively small-scale attacks on their compounds. Our approach is to detect moving objects in a wide area around the compound. This system was successfully demonstrated at the TNO facility in The Netherlands. 
The monitoring concept is illustrated by the SOWNet system in figure 2. There are two sensor-node groups. The first group consists of 50 nodes (TNOdes) equipped with passive infrared sensors. The nodes are tactically deployed over an area of 100 meters around the compound. The nodes are able to detect movements, assess the level of danger and if necessary they can send a signal with position information to the second group, the camera group. The camera is able to turn $360^{\circ}$, implying that it can observe all sub-areas around every node in the network. For the human interfacing, a monitoring station was added (PDA/laptop) for displaying status messages, node information and camera images. Hence, in a real operation, the operators can verify the level of the threat from a safe distance and decide which action must be taken.

The sensor-nodes are encased in PVC-pipes (see figure 3), thus creating a lightweight, mobile and robust monitoring system. Deployment of the network is easy; the pipes are pushed into the ground. On the top of each pipe, a magnet is mounted. In the pipes, a reed-switch is attached and used as power-switch. So when the nodes are pushed down, they are powered. Once achieved, they automatically join the sensor network.

As shown in figure 2, the network topology in the system takes the form of a decentralized network. The network is divided into several clusters. Each cluster has a gateway, or cluster master, which can communicate to other cluster masters. One cluster master is assigned to communicate with the base station. In the current demonstration system, the position of each node is hard-coded with respect to the position of its cluster (automatic positioning is a difficult aspect in ad-hoc sensor networks [3]). In case a sensor detects motion, an alarm signal is sent by that node to the camera-node group. After an alarm message has been received, the camera(s) automatically turn to the specific cluster. In this manner, the operator gets an overall view of the cluster area and may manually zoom-in if desired.

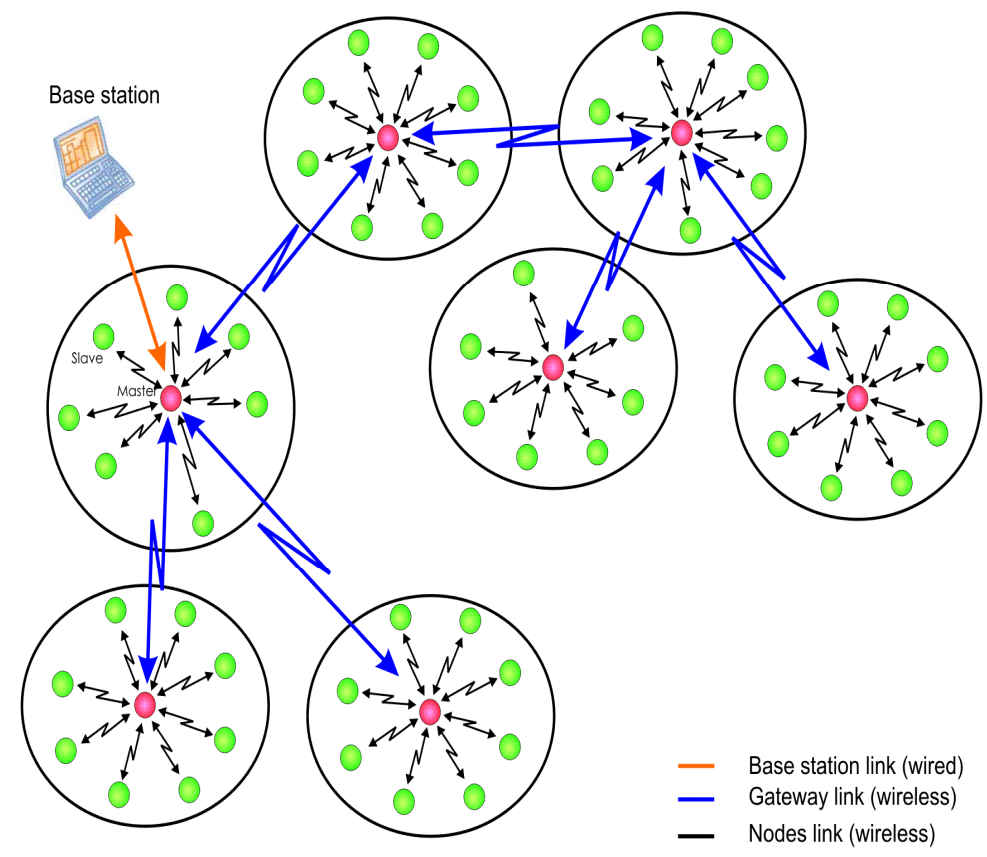

Figure 2. Sensor nodes configuration of the SOWNet System. 


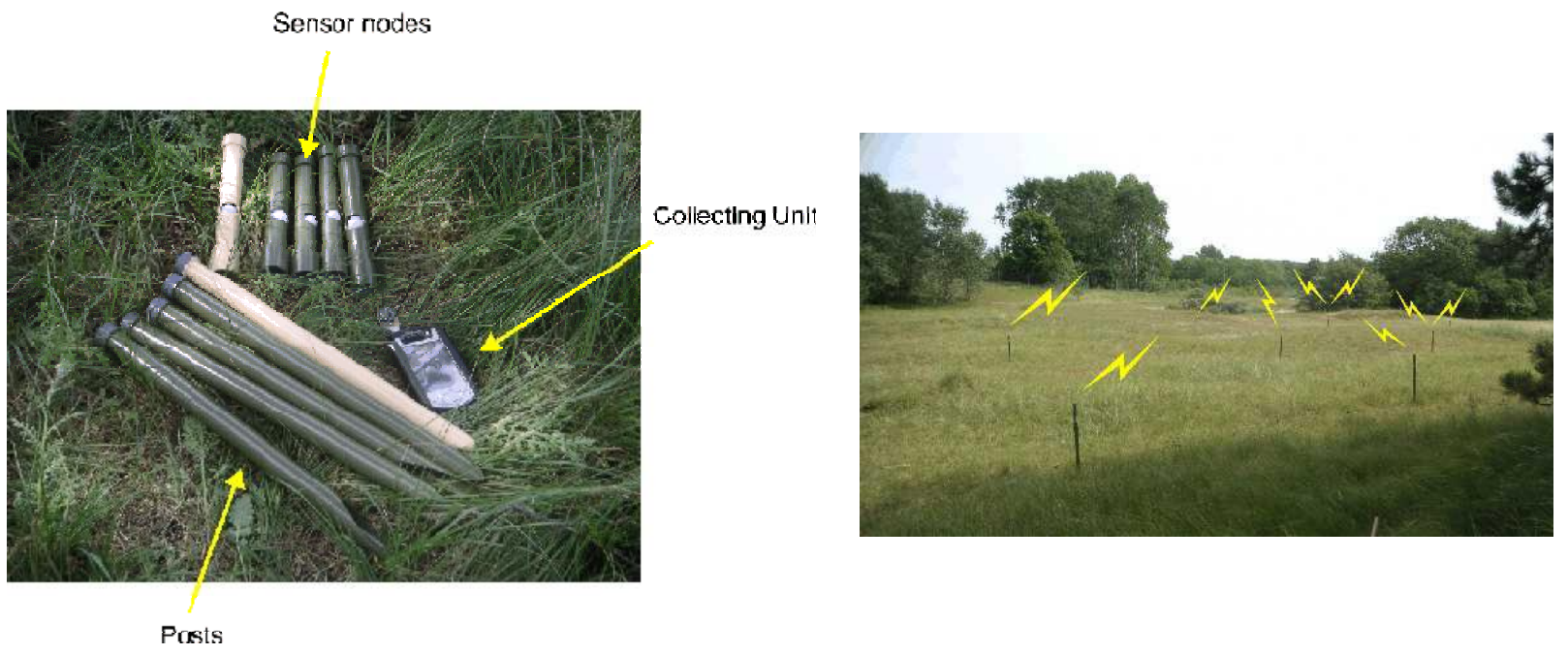

Figure 3. Physical sensor nodes (left). The nodes were used in the compound security demonstration system to track persons in a field (right).

\section{WIRELESS BUOY NETWORKS}

So far, the argument has been about land-based wireless sensor networks. In this section, we will elaborate on the potential use of such networks in a maritime setting. Although floating sensing buoys are not new, most existing systems are rather static. That is, they are not part of an independent, ad-hoc network, neither are they self-propelled. For example the National Data Buoy Center (NDBC) from the National Oceanic and Atmospheric Administration (NOAA) of the United States, designs, develops, operates, and maintains a network of data collecting buoys [6]. The buoys measure atmospheric and oceanographic variables, such as air and water temperature, sea level and wind speed. They are placed all over the world and are used to detect abnormal conditions, such as a storm or a tsunami. The buoys are anchored to a specific location. Their data is sent to a data-collection center where it is made accessible to users through the internet. Though interesting for weather observation, for area monitoring more buoys would be required. Hence, we are looking into easy-deployable, preferable mobile, floating wireless sensor suites. Such intelligent buoys could take a shape as shown in figure 4 . The different components of the intelligent buoy are:

- Wireless communication (through air). The buoys can communicate with each other by means of wireless communication. For this reason they are equipped with a data-communication link. The transmission power and antenna length must be proportioned such that waves do not influence the quality of transmission.

- Sensors. The type of sensor(s) depends on the task of the buoy(s). In principle, all buoys will be equipped with sonar for detecting submerged objects and acoustic sources. Furthermore, a GPS receiver for positioning monitoring will be included. Other sensors may include temperature, wave heights, wind speeds, depth, etc. 
- Intelligent computing. When a lot of data is collected by the buoys, it is not efficient to send all the data to another buoy or to a central station. In such a case, local signal processing is often preferred. Furthermore, signals from neighboring buoys can be combined to get better situational awareness, even before sending it to other places.

- Propulsion/steering. The buoys must be able to navigate in order to ensure the appropriate monitoring position. In particular inter-distance between the buoys can be well organized in this manner. For that purpose the buoys need a pod containing battery, a motor-rudder and a motor driving the propulsor. It is clear that the buoys will have restricted mobility as their battery capacity will be limited. The buoys therefore, must be deployed close to their intended operational area.

- Mechanisms for dispatch and retrieval. The buoys will have to be put out from ships (or airplanes). After use they need to be picked up again. For this, mechanical means need to be developed to enable such handling of the buoys.

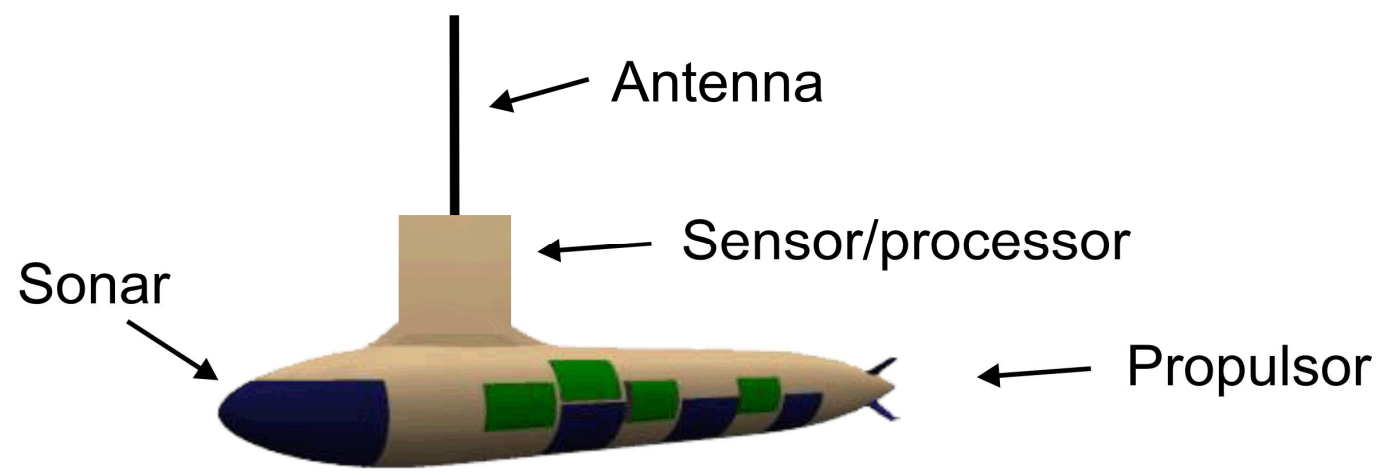

Figure 4. A prototype of an intelligent (mobile) buoy.

\subsection{Applications of buoy networks}

In this section, typical applications of buoy networks will be discussed. The discussion is taken from a military stance, hence much attention is paid to security and protection.

\subsubsection{Area protection}

The most obvious task for a (mobile) buoys network is surveillance of certain areas to support protection. In particular harbors and bay areas are vulnerable targets of attacks and must be safe-guarded. Intelligent buoys will be easy and cheap to deploy compared to using surveillance ships. A typical deployment of networked buoys would be to have many floating around in order to detect any movement as well as size and direction of all moving objects in the area. The tracks of the moving objects are then reported to a control station.

After an object has been detected, it is required to assess its identity. A buoy network could be helpful by analyzing its movements and deliver a first rude threat assessment (e.g., to determine whether it is a tourist or a terrorist on a jet ski). Also divers are a serious threat to naval forces as they can approach covertly below the water surface. Buoys equipped with sonar devices could detect such activity. Note that there is a large trade-off between false alarm rate and risk acceptance for such tasks. 


\subsubsection{Ship protection}

When a ship is at anchor in an assumed friendly environment, usually the ship's engine is shut down and most (observation) systems are powered off to save energy. During that time the ship is vulnerable to (asymmetric) enemy attacks, which may come from:

- Above water - unmanned: like a buoy or a raft;

- Above water - manned: like a jet ski, swimmer or a propelled boat;

- Underwater - unmanned: like an unmanned underwater vehicle (UUV), or a mine;

- Under water - manned: like small submarines or a diver.

A ring of intelligent buoys that autonomously form a sensing network at the water is an appropriate approach to help protect the ship(s). The buoys would place themselves in the area optimally with respect to each other and the object to protect. In Figure 5, an example is shown in which the buoy networks adopt the shape of several rings around the ship. The sensors can be deployed in various ways. They could be put around the ship by sailors, or dropped by an aeroplane, fired from a ship or shore or covertly positioned in advance, for example by a submarine (for a profound description of ship-protection see [5]).

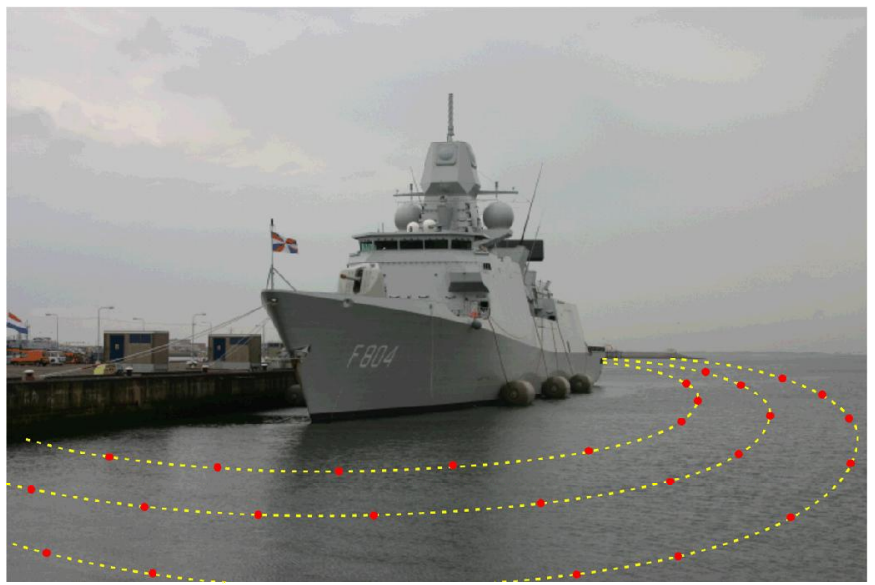

Figure 5. A set of (small) buoys can be deployed around the ship as detection and identification rings. 


\section{CONCLUSION}

Wireless sensor networks provide an innovative computing paradigm facilitating distributed information fusion. By exploiting many small and cheap wireless sensor nodes, a flexible computing network can be created. The work at TNO focuses on perfecting this technology, which is currently based on the SOWNet ${ }^{\mathrm{TM}}$ platform and TNOdes ${ }^{\mathrm{TM}}$ sensor-nodes. $^{\text {. }}$. Hence all the crucial aspects that are related to sensor networks, such as life-time of a sensor-node and reliability of the system are studied, developed and tested extensively. The aim of this research is to provide (military) forces with systems than can support the safety of personnel and equipment.

The presented demonstration system backs our view that soon sensor networks will be used in real military operations. In cooperation with the Dutch military, TNO develops the sensor networks technology further to provide the military with the desired improved situational awareness.

As mentioned, land-based sensor networks are widely being developed. In this paper we also discussed the use of such networks in a maritime environment. It appears that the sensor nodes (buoys) may be larger and more expensive than the land-based ones, given their specific requirements with respect to buoyancy and range, in particular if they need to be mobile. Nevertheless, we observe an emerging need for water-based smart networked systems to support in the continuous (unmanned) protection of areas and equipment. The next step is to develop a truly deployable networked buoy.

Although the focus is on military applications, note that many civil applications can benefit from this technique. Currently identified applications include precision agriculture, dyke protection and monitoring \& control in the process industry.

\section{ACKNOWLEDGEMENT}

The authors would like to thank Maarten Ditzel as well as the electro-mechanical workshop from TNO for their support in creating the TNOdes. This work was carried out within program V219 of the Dutch military defense department.

\section{REFERENCES}

1. D. Culler, D. Estrin and M. Srivastava. Overview of Sensor Networks. In IEEE Computer, August 2004.

2. T. Yan, T. He, and J. A. Stankovic. Differentiated Surveillance for Sensor networks. First ACM Conference on Embedded Networked Sensor Systems (SenSys 2003), Los Angeles, CA 2003.

3. K. Langendoen and N. Reijers. Distributed Localization in Wireless Sensor Networks: A Quantative Comparison. Computer Networks (Elsevier), special issue on Wireless Sensor Networks, November 2003.

4. J.A.A.J. Janssen and Maris, M.G. Self-Configurable Distributed Control Networks on Naval Ships. Proceedings of the Thirteenth International Ship Control Systems Symposium. Orlando, FL, 2003.

5. W. Kavelaars, B. Dunnebier and P. Vierveijzer. Wireless Sensor Networks in Naval Force Protection. In Naval Forces, 2005.

6. http://www.ndbc.noaa.gov 